\title{
A Model for Clinical Informatics Education for Residents: Addressing an Unmet Need
}

\author{
Mark V. Mai ${ }^{1,2}$ Brooke T. Luo ${ }^{1}$ Evan W. Orenstein ${ }^{1,2}$ Anthony A. Luberti ${ }^{1,2}$ \\ ${ }^{1}$ Department of Pediatrics, Children's Hospital of Philadelphia, \\ Philadelphia, Pennsylvania, United States \\ 2 Department of Biomedical and Health Informatics, Children's \\ Hospital of Philadelphia, Philadelphia, Pennsylvania, United States \\ Address for correspondence Mark V. Mai, MD, Children's Hospital of \\ Philadelphia, 2716 South Street, Ste 15-194, Philadelphia, PA 19146, \\ United States (e-mail: maimv@email.chop.edu).
}

Appl Clin Inform 2018;9:261-267.

Abstract

Keywords

- medical informatics

- medical education

- internship and residency

- strategies for health IT training
Opportunities for education in clinical informatics exist throughout the spectrum of formal education extending from high school to postgraduate training. However, physicians in residency represent an underdeveloped source of potential informaticians. Despite the rapid growth of accredited fellowship programs since clinical informatics became a board-eligible subspecialty in 2011, few resident physicians are aware of their role at the intersection of clinical medicine and health information technology or associated opportunities. In an effort to educate and engage residents in clinical informatics, Children's Hospital of Philadelphia has developed a three-pronged model: (1) an elective rotation with hands-on project experience; (2) a longitudinal experience that offers increased exposure and mentorship; and (3) a resident founded and led working group in clinical informatics. We describe resident participation in these initiatives and lessons learned, as well as resident perceptions of how these components have positively influenced informatics knowledge and career choices. Since inception of this model, five residents have pursued the clinical informatics fellowship. This educational model supports resident involvement in hospital-wide informatics efforts with tangible projects and promotes wider engagement through educational opportunities commensurate with the resident's level of interest.

\section{Background and Significance}

The demands and challenges facing biomedical and clinical informatics highlight the need for the development of a pipeline of future informaticians who can bridge the complex issues of clinical medicine with the innovative solutions of information technology. Already, opportunities for education in clinical informatics are becoming more prevalent throughout the spectrum of medical training. The American Medical Informatics Association (AMIA) High School Scholars Program pairs accepted high school students with a mentor to work on a biomedical informatics project and present at the AMIA Annual Symposium. ${ }^{1}$ At the University of Pittsburgh, high school students have been exposed to biomedical and pathology informatics through an 8-week summer course since 2009 in anticipation of the escalated demand for informaticians. ${ }^{2}$ Increasingly, college courses are incorporating a discussion of information sciences into biological and health care-related topics. ${ }^{3-5}$ With support from the National Library of Medicine, Masters and PhD-level graduate school programs in biomedical informatics have been particularly well developed. ${ }^{6-8}$ Many medical school curricula include a discussion of the role of information technology in medicine. ${ }^{5,9-11}$ Following residency, physicians have the opportunity to engage in further informatics training through the clinical informatics fellowship or the practice pathway. ${ }^{12-15}$ However, there exists a gap of clinical informatics training at the residency level. received

December 19, 2017

accepted after revision

March 10, 2018
Copyright (c) 2018 Schattauer 
While tapping into this group can be fraught with challenges due to residents' intense clinical obligations, residents are uniquely positioned to be receptive to and contribute to the field of informatics. As front-line clinicians, residents are a key demographic of electronic health record (EHR) users whose daily workflow is directly impacted by the structure of the EHR. Residents are intimately aware of a system's strengths and have a vested interest in fixing shortcomings at the sharp end of care delivery. They represent a potential source of collaborative solutions and practical feedback. Because of the nature of their training, residents are also well acquainted with the intricacies of various workflows including people and processes in a given institution. Residents represent a large proportion of the workforce at many hospitals, increasing the points of contact for collaboration. Furthermore, as a result of the opportunities prior to residency, as previously described, recent generations of residents are increasingly adept with the technologies used in clinical informatics, although their familiarity with the principles may be lacking. Engagement of resident physicians in hospital informatics efforts can accelerate implementation of meaningful improvements, while preparing residents to become proficient physicians in the electronic era.

Based on traditional models of clinical subspecialty fellowships, the majority of applicants to clinical informatics fellowships are likely to come directly from residency training. To provide a glimpse into potential fellowships, programs require several clinical rotations that provide in-depth contact with core principles, daily activities, and potential mentors in the field. A rotational correlate does not exist at most training institutions for clinical informatics, although some have successfully established medical student and resident electives. ${ }^{16-18}$ Until recently, little had been published on the education of residents in the field of medical informatics since $1999 .^{19}$ In 2017, the University of California, Los Angeles (UCLA) Health Informatics program shared their approach consisting of a longitudinal program where accepted residents and fellows follow a yearlong curriculum covering major topics in clinical informatics, learn to develop new tools in the EHR, and partner with a mentor to develop and implement an informatics project. $^{20}$ Comprehensive programs like these improve the visibility of clinical informatics as a potential career option for trainees. However, this approach required substantial resources, including monthly lectures, an application acceptance program, and a monetary project stipend after completion of the program. Scaling up these educational initiatives in this critical period may be difficult without such resources.

In this article, we present a model of a clinical informatics curriculum that has been in place at Children's Hospital of Philadelphia, a large urban academic pediatrics hospital, since 2012. The pediatrics residency program consists of 163 residents. The Department of Biomedical and Health Informatics supports a clinical informatics fellowship with 4 fellows and 10 board-certified faculty educators. This model for resident education in clinical informatics includes options such as an introductory elective, a longitudinal experience, and a resident working group. We also present residents' perceptions of the impact of different curricular elements, as well as their career trajectories after elective participation.

\section{Key Components of Informatics Educational Model}

During the 3-year pediatric residency training program, residents have exposure to informatics education at multiple levels. These range from required experiences for all residents to optional, short- and long-term informatics educational opportunities (-Table 1 ). The central pillars of the model include a health informatics elective, a longitudinal experience, and a resident working group.

\section{Short-Term Experience-Health Informatics Elective}

The health informatics elective combines structured didactics with skill-building online EHR training modules. The elective is 2 to 4 weeks in duration and offered throughout the year. Participants in the elective meet with the course director prior to or at the start of the elective to assess the participant's knowledge and proficiency in health informatics to personalize their goals and objectives for the rotation. This approach aims to provide a foundation of informatics knowledge, but emphasizes exploration within the individual resident's interest area. Activities include viewing online recorded lectures, reading select textbook chapters and journal articles, and participating in various

Table 1 Resident experiences in clinical informatics

\begin{tabular}{|c|c|c|}
\hline & Curricular & Extracurricular \\
\hline Required & $\begin{array}{l}\text { - EHR case simulations }{ }^{23} \\
\text { - Introductory informatics lecture } \\
\text { - Knowledge management lecture }\end{array}$ & - EHR efficiency workshops \\
\hline Optional (short-term) & - Elective rotation & $\begin{array}{l}\text { - Mentorship by informatics faculty, fellows, staff, and peers } \\
\text { - Informatics committee meetings } \\
\text { - Invited lecturers } \\
\text { - Regional and national informatics conferences }\end{array}$ \\
\hline Optional (long-term) & - Longitudinal experience & $\begin{array}{l}\text { - Resident informatics working group } \\
\text { - Independent project }\end{array}$ \\
\hline
\end{tabular}

Abbreviation: EHR, electronic health record. 
informatics-related meetings and conferences (see sample objectives and activities in -Appendix Tables A1-A4). In addition to meeting with other faculty, participants continue to meet weekly with the course director to follow-up on progress and to adjust their activities to meet their individualized objectives. For each resident in the elective, this structure requires approximately 2 hours per week of time commitment from the course director as well as approximately 2 hours per week of ad hoc meetings with other faculty members or Information Services (IS) staff with expertise in the resident's area of interest.

During the elective, residents are encouraged to complete projects, providing hands-on experience where they apply newfound informatics knowledge to clinically relevant scenarios. The course director and the participant specify a project that will be feasible within their time commitment, that will be appropriately mentored, and that will align with their personalized goals and objectives. Depending on the resident's interest, their contribution to the project may be limited to the duration of the rotation or continue to evolve after the elective. Of note, nearly all successful projects were started during the elective and then benefitted from crowdsourcing through the resident working group described below.

As part of their projects, elective participants may be given access to an EHR sandbox development environment, which does not migrate to the production environment. This environment exists as a space to test new functionality without disrupting operational processes. Residents are required to complete a set of training modules hosted by the EHR vendor ( -Appendix Table A3) in addition to a 1-hour review of best practices with the informatics course director. The course director subsequently works with our IS department to provide the resident with sandbox development environment access. Residents are expected to use this access to augment their skills in other core competencies, like performing workflow analyses, understanding the impact of EHR build on patient care, and aligning user needs with EHR capabilities. Residents have the option of presenting their work to a hospital committee, who may then decide to migrate their work to production. Aside from time invested by the course director and that of IS, there is no additional cost associated with providing residents with this access.

\section{Longitudinal Experience}

All pediatric residents at Children's Hospital of Philadelphia have the option of applying to participate in a longitudinal track, which allows residents to increase their exposure to academic domains outside of traditional clinical experiences. Examples include bioethics, health care disparities, as well as clinical informatics. Residents in this informatics track engage in advanced didactics (both directed and selfguided), meet with faculty mentors throughout the remainder of their training, attend committee meetings, and work on a long-term project with goal of completion prior to graduation from residency. At graduation, this advanced skill participation is acknowledged on their diploma along with the participant's training in pediatrics.

\section{Resident Informatics Working Group}

Following the establishment of the formal educational curriculum, a group of residents interested in workflow improvement started a resident informatics working group in 2013. This group, also known as the New EHR Resident Development (NERD) Squad, meets in the evenings every 1 to 2 months to discuss resident workflow issues with potential EHR solutions. As part of the NERD Squad, residents are encouraged to pursue the elective in health informatics and offered a wide array of opportunities to be involved in hospital initiatives. Faculty and fellows within clinical informatics also routinely attend meetings, providing mentorship and guidance on core informatics principles. These meetings often take place in the evening following resident shifts with funding for meals provided by the clinical informatics department.

The NERD Squad serves as a forum for nontechnically oriented residents to voice their concerns. As a result, it creates an informal space for crowdsourcing, accelerating the incorporation of user input in the design process of residentled projects. Example projects resulting from collaborations of residents with informatics faculty are detailed in -Table 2 . The majority of resident efforts has involved enhancing documentation support and peer education. All content created by residents requires a faculty mentor to review before presentation at an EHR operational meeting. Following approval, the content is incorporated into standard change control processes.

\section{Example Resident Project}

The following example of a resident-led project serves to highlight the interplay between the informatics elective, longitudinal experience, mentors, and resident working group. Previously, residents were required to free-text all discharge instructions once a patient was ready for discharge contributing to documentation burden and variation in discharge care for common diagnoses. During the clinical informatics elective, one pediatric intern proposed

Table 2 Examples of resident projects

\begin{tabular}{|l|}
\hline Project \\
\hline Handoff templates for common admission diagnoses \\
\hline Discharge summary templates for common diagnoses \\
\hline $\begin{array}{l}\text { Standardized inpatient discharge instructions for common } \\
\text { diagnoses }\end{array}$ \\
\hline $\begin{array}{l}\text { Documentation template for medically complex foster } \\
\text { children }^{\text {a }}\end{array}$ \\
\hline Templated list of common contingency plans \\
\hline EHR simulation curriculum for interns \\
\hline EHR training sessions \\
\hline Health hackathon \\
\hline
\end{tabular}

Abbreviation: EHR, electronic health record.

${ }^{a}$ Initiated and completed within a 4-week elective; all other projects were initiated during an elective but completed in conjunction with the resident working group. 
reviewing the top 20 admission diagnoses to prioritize creation of standard templates. In conjunction with faculty mentorship from clinical informatics, she drafted several templates and incorporated feedback from the resident working group and clinical faculty. After implementation into the EHR production environment, she presented this work to the resident working group, and other interested residents began adding content. These contributions expanded the number of discharge diagnoses and grew into a longitudinal project for the original resident. This experience allowed the resident to learn key informatics principles in workflow analysis, change management, and stakeholder engagement.

\section{Participation}

Pediatrics residents have one elective block during their first year, two during their second year, and four during their third year. The incoming resident cohort ranges from 48 to 54 residents. Since the resident health informatics elective was established in 2012, 23 residents have completed the elective, of whom 4 (17\%) pursued further education through the longitudinal experience, and 10 (43\%) participated in the resident working group. From each residency class, on average, $5 \%$ of residents participated in the elective. An additional 33 residents have participated in the resident working group without engaging in other informatics opportunities.

\section{Resident Perceptions}

We contacted all 23 prior participants of the health informatics elective for feedback on how components of the educational model influenced their informatics knowledge and career choices ( - Fig. 1). Although this was not a formal evaluation of the model, many elective participants also interacted with other components of the educational model including the longitudinal experience, the resident working group, as well as mentorship activities and a clinical informatics project. Among the 19 respondents, 79\% (15/19) found the elective to be very or extremely influential on their knowledge base and 60\% (6/10) who had completed residency and were still involved in informatics felt similarly about the elective's impact on their career choice. In addition to the elective, the majority of respondents highlighted that participation in a clinical informatics project, mentorship activities, and the resident working group were similarly influential on their knowledge base and career choices.

\section{Career Trajectories}

Following residency, five elective participants went on to pursue additional training through the clinical informatics fellowship. From those who provided feedback, all 14 postresidency participants indicated some continued involvement in clinical informatics or related activities, with 4 of 14 (29\%)
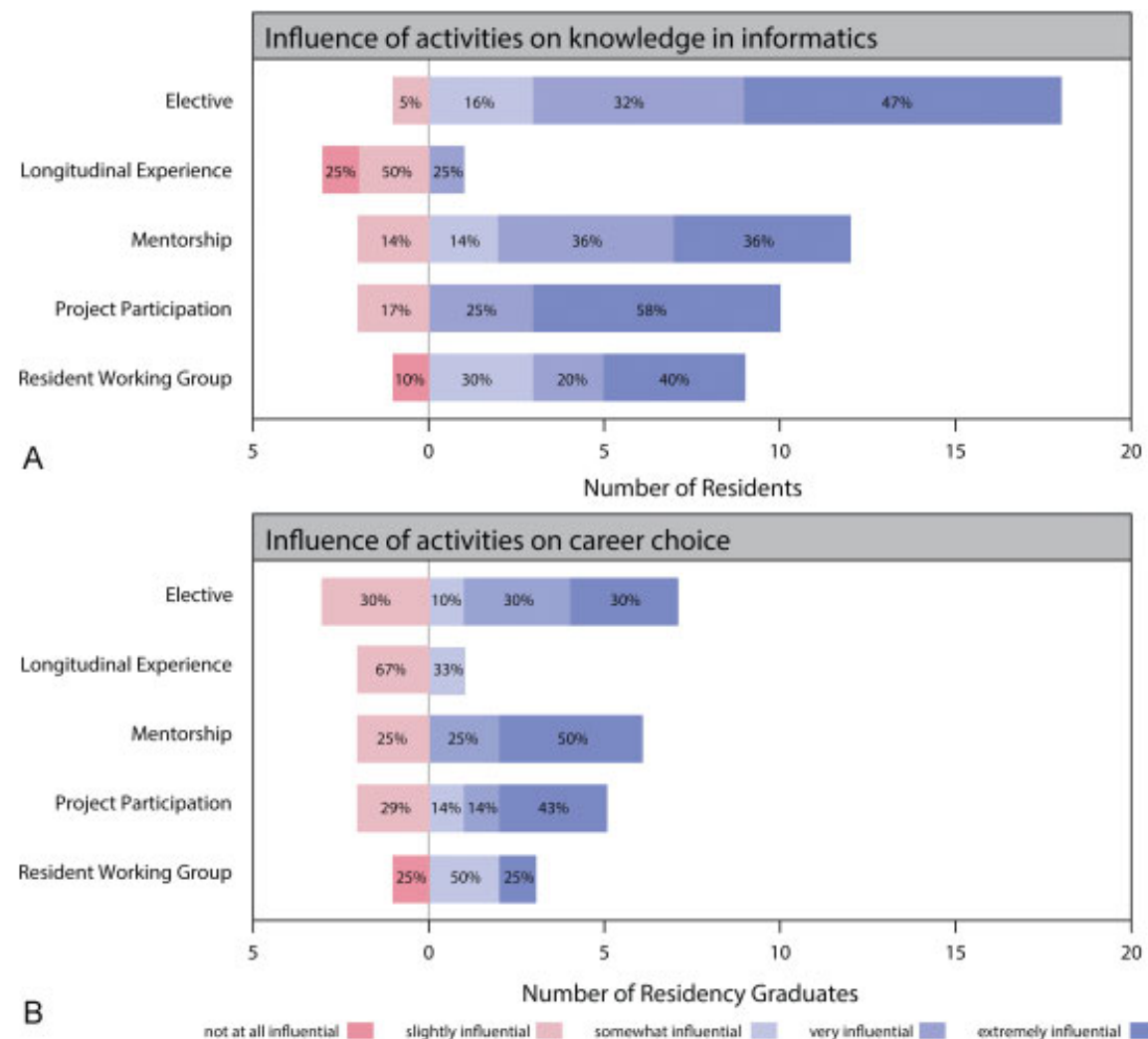

Fig. 1 Factors influencing residents' knowledge of clinical informatics and residency graduates' career choices. 
acting as an "EHR clinical champion," 3 of 14 (21\%) participating in a clinical decision support committee, 8 of 14 (57\%) participating in informatics related research, 8 of 14 (57\%) incorporating informatics into quality improvement work, and 6 of 14 (43\%) acting as informatics educators.

\section{Discussion}

Using a model focused on resident education, project experience, and a grassroots community, we have improved resident engagement in clinical informatics at our institution. This approach has led to organizational benefits in the form of tangible projects and increased front-line provider participation in clinical informatics efforts. Additionally, residents have noted that these educational opportunities influenced their knowledge in clinical informatics and career choices, with all participants incorporating informatics training into their careers in some capacity and a growing number of residents choosing to specialize in clinical informatics.

Residency training represents a critical window of opportunity for the education and recruitment of future physicians into the field of clinical informatics. However, residents have very little free time outside of clinical duties, limiting chances for exposure to clinical informatics without a prior interest. One of the key lessons learned as we have developed this educational model is that wider engagement can be achieved through educational opportunities commensurate with the resident's level of interest. For residents without previous experience, the resident working group offers exposure to clinical informatics in a social setting without necessitating the commitment of an elective. For those who want to learn more, the health informatics elective provides residents an opportunity to increase their knowledge base, to meet mentors, and to lead a project. As residents gain a deeper interest, the longitudinal experience offers a chance for advanced skill development throughout residency. This approach meets the individualized educational needs of residents with varying levels of interest. Since inception of this educational model, five participants are pursuing clinical informatics fellowship training, suggesting that this investment may grow the applicant pool to fill an unmet clinical need. ${ }^{21}$

The descriptive discussion was not intended to prove that the organizational benefits and increased resident engagement were caused by the educational model presented here. The feedback solicited from previous elective participants was not gathered from a validated scientific instrument, but rather was intended to inform whether residents perceived the components of the educational model as influential on their clinical informatics knowledge and career trajectories. We also did not assess this model compared with alternatives. Nonetheless, in the setting of these educational opportunities, we have seen a sharp increase in awareness of clinical informatics within the residency program, the number of interested residents pursuing additional training, the impact of resident-led projects, and the participation of residents in operational informatics initiatives. Additionally, while we anticipate that this model would be generalizable to other settings, our institution benefits from facilitators such as a large number of clinical informatics faculty and clinical champions, a department focused on informatics and education, and a fellowship program.

To our knowledge, the only other published informatics curriculum focused on residents is the UCLA Health Resident Informatics Program, where moderate investments in an application process, didactic training, a project stipend, and faculty mentorship have led to impressive accomplishments and resident engagement. ${ }^{20}$ In contrast, our model requires fewer funding resources to support the resident working group, although faculty time for mentorship and meetings remains critical. Nonetheless, we have seen growing interest in resident-led activities through the working group despite concerns about resident workload and training gaps. ${ }^{22}$

\section{Conclusion}

Clinical informatics is increasingly permeating into the practice of medicine. Residency is a vital time to educate physicians about the field of clinical informatics to support their clinical work and to inspire the next generation of leaders. Our institution has created a combination of shortterm and longitudinal opportunities to expose residents to the field. This exposure has in turn led to a cultural shift, resulting in the dissemination and spread of informatics knowledge within the residency program and has generated a pipeline of informatics fellowship applicants. Even among residents who did not pursue an advanced degree in clinical informatics, all postgraduate residents now incorporate clinical informatics in their careers in some capacity. While we cannot distinguish the impact of this educational model from increasing familiarity with technology, elective participants indicate that these educational experiences influenced their knowledge and career choices. We have also seen tangible organizational benefits from projects initiated during electives, supported by a resident working group, and incorporated into longitudinal experiences. Expansion of this and similar models to promote resident engagement in clinical informatics across training programs has the potential to accelerate healthcare improvements and develop the clinical informaticians of the future.

\section{Clinical Relevance Statement}

Opportunities for clinical informatics education extend from high school to beyond clinical fellowship training, but a gap exists during residency. We describe a low-resource, but potentially high-impact educational support structure that appears to have influenced not only participants' knowledge of clinical informatics, but also their future career activities. Similar models to promote resident engagement in clinical informatics have the potential to accelerate health care improvements and develop the clinical informaticians of the future. 


\section{Multiple Choice Question}

During which stage of medical training is there a clear gap in informatics education?
a. High school
b. Medical school
c. Residency
d. Fellowship
e. Postfellowship

Correct Answer: The correct answer is option c. Opportunities for education in clinical informatics are becoming more prevalent throughout the spectrum of medical training. The AMIA High School Scholars Program pairs accepted high school students with a mentor to work on a biomedical informatics project and present at the AMIA Annual Symposium. Increasingly, college courses are incorporating a discussion of information sciences into biological and health care-related topics. Masters and PhD-level graduate school programs in biomedical informatics have been particularly well developed. Many medical school curricula include a discussion of the role of information technology in medicine with some incorporating biomedical and clinical informatics. Following residency, physicians have the opportunity to engage in further informatics training through the clinical informatics fellowship or the practice pathway. However, there exists a gap of clinical informatics training at the residency level, as most institutions do not offer an elective rotation in clinical informatics.

\section{Protection of Human and Animal Subjects}

Collection of resident feedback and perceptions was deemed exempt by the Institutional Review Board at Children's Hospital of Philadelphia.

\section{Conflict of Interest}

None.

\section{Acknowledgments}

We would like to thank the faculty of Children's Hospital of Philadelphia for contributing their time and mentorship of our residents. We would also like to thank Naveen Muthu for providing feedback on early versions of the manuscript. Finally, we would like to acknowledge members of the NERD Squad for their continuing support, feedback, and friendship.

\section{References}

1 Unertl KM, Finnell JT, Sarkar IN. Developing new pathways into the biomedical informatics field: the AMIA High School Scholars Program. J Am Med Inform Assoc 2016;23(04):819-823

2 Dutta-Moscato J, Gopalakrishnan V, Lotze MT, Becich MJ. Creating a pipeline of talent for informatics: STEM initiative for high school students in computer science, biology, and biomedical informatics. J Pathol Inform 2014;5(01):12
3 Foy D, Canfield K, Schwartz J. Undergraduate health care informatics education: a needs analysis and proposed curriculum. Proceedings Symp Comput Appl Med Care 1994:37-40

4 Hasman A, Haux R. Curricula in medical informatics. Stud Health Technol Inform 2004;109:63-74

5 Hasman A, Mantas J, Zarubina T. An abridged history of medical informatics education in Europe. Acta Inform Med 2014;22(01): 25-36

6 Hersh WR. The full spectrum of biomedical informatics education at Oregon Health \& Science University. Methods Inf Med 2007;46 (01):80-83

7 Braude RM. A descriptive analysis of National Library of Medicinefunded medical informatics training programs and the career choices of their graduates. Med Decis Making 1991;11(01):33-37

8 Hoffmann S, Ash J. A survey of academic and industry professionals regarding the preferred skillset of graduates of medical informatics programs. Stud Health Technol Inform 2001;84(Pt 2):1028-1032

9 Hart JK, Newton BW, Boone SE. University of Arkansas for Medical Sciences electronic health record and medical informatics training for undergraduate health professionals. J Med Libr Assoc 2010;98(03):212-216

10 Silverman H, Cohen T, Fridsma D. The evolution of a novel biomedical informatics curriculum for medical students. Acad Med 2012;87(01):84-90

11 Banerjee R, George P, Priebe C, Alper E. Medical student awareness of and interest in clinical informatics. J Am Med Inform Assoc 2015;22(e1):e42-e47

12 Longhurst CA, Pageler NM, Palma JP, et al. Early experiences of accredited clinical informatics fellowships. J Am Med Inform Assoc 2016;23(04):829-834

13 Detmer DE, Munger BS, Lehmann CU. Clinical informatics board certification: history, current status, and predicted impact on the clinical informatics workforce. Appl Clin Inform 2010;1(01):11-18

14 Silverman H, Lehmann CU, Munger B. Milestones: critical elements in clinical informatics fellowship programs. Appl Clin Inform 2016;7(01):177-190

15 Baldwin K, Bell DS. ACGME Clinical Informatics Fellowships Lessons Learned. In: AMIA 2016 InSpire Conference Proceedings; 2016

16 Partners Clinical Informatics Elective. Available at: http://informatics.partners.org/elective-rotation/. Accessed December 19, 2017

17 Harvard Medical School Medical Student Clinical Informatics Elective. Available at: http://www.medcatalog.harvard.edu/coursedetails.aspx?cl=\&id=19532. Accessed December 19, 2017

18 Stanford medical student elective. Available at: http://bmi.stanford.edu/biomedical-informatics-students/electives.html. Accessed December 19, 2017

19 Jerant AF. Training residents in medical informatics. Fam Med 1999;31(07):465-472

20 Singer JS, Cheng EM, Baldwin K, Pfeffer MA; UCLA Health Physician Informaticist Committee. The UCLA Health Resident Informaticist Program - a novel clinical informatics training program. J Am Med Inform Assoc 2017;24(04):832-840

21 Lucey CR. Is medical education a public or a private good? Insights from the numbers. JAMA 2017;318(23):2303-2305

22 Luo B, Luberti AA, Orenstein E, Mai MV. Decentralizing Innovation: The Benefits of an Informatics Curriculum that Provides EHR Build Experience to Empower Residents. In: AMIA 2017 InSpire Conference Proceedings; 2017

23 Orenstein E, Rasooly I, Phillips W, et al. The Impact of Simulation on Electronic Health Record Use Patterns among Pediatric Residents. In: AMIA 2017 Annual Symposium Proceedings; 2017:153-154 


\section{Appendix}

An Example of an Individualized Curriculum for the Health Informatics Elective for a Resident Interested in Usability

Appendix Table A1 Sample learning objectives

1. State and understand how the discipline of clinical informatics intersects with and influences the three domains of clinical care, local and national health care systems, and information and communications technologies

2. Describe and understand the role of a clinician in applying the principles of health informatics in the daily practice of pediatrics

3. Participate in simple usability projects with faculty mentors in health informatics focused on optimization of electronic health record systems

4. Facilitate discussions related to the ongoing improvements of clinical information systems as a "clinician champion" or liaison between the clinical informatics team and your clinical peers
Appendix Table A3 EHR build modules

\begin{tabular}{|l|l|l|}
\hline Topic & $\begin{array}{l}\text { Number of } \\
\text { videos }\end{array}$ & Duration \\
\hline Building note templates & 3 videos & $25 \mathrm{~min}$ \\
\hline Building order sets & 3 videos & $42 \mathrm{~min}$ \\
\hline Building alerts & 2 videos & $28 \mathrm{~min}$ \\
\hline
\end{tabular}

Abbreviation: EHR, electronic health record.

Appendix Table A4 Sample activities during elective

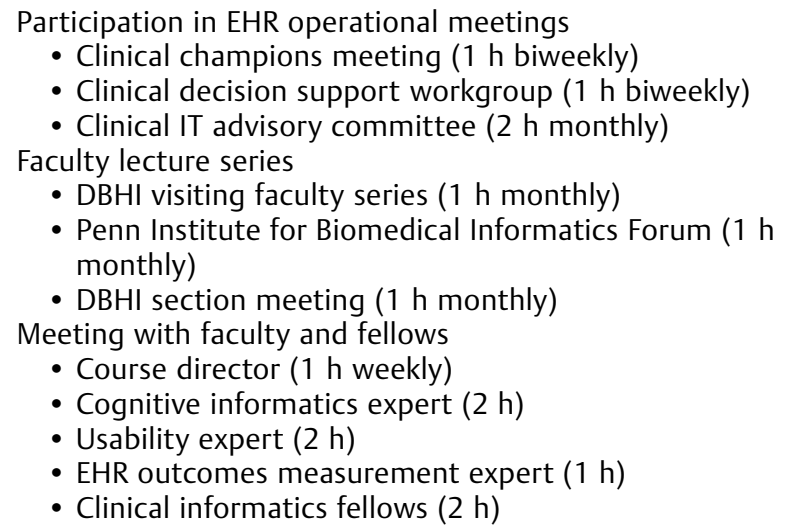

Abbreviations: DBHI, Department of Biomedical and Health Informatics; EHR, electronic health record; IT, information technology.

Appendix Table A2 Didactic materials

Selected readings for all elective participants

- Selected chapters from: Shortliffe EH and Cimino JJ. Biomedical Informatics: Computer Applications in Health Care and Biomedicine, 2006

- Ash JS, Stavri PZ, Dykstra R, Fournier L. Implementing computerized physician order entry: the importance of special people. Int J Med Inform. 2003;69(2-3):235-250

- Weed LL. Medical records that guide and teach. N Engl J Med. 1968 Mar 14;278(11):593-600

- Bates DW, Kuperman GJ, Wang S, Gandhi T, Kittler A, Volk L, et al. Ten commandments for effective clinical decision support: making the practice of evidence-based medicine a reality. J Am Med Inform Assoc. 2003;10(6):523-530

- Han YY, Carcillo JA, Venkataraman ST, Clark RSB, Watson RS, Nguyen TC, et al. Unexpected increased mortality after implementation of a commercially sold computerized physician order entry system. Pediatrics. 2005 Dec;116(6):15061512

For an example resident interested in usability

- Selected chapters from: Zhang J, Walji M. Better EHR, Usability, workflow and cognitive support in electronic health records. 2014

- Selected chapters from: Patel VL, Kannampallil TG, Kaufman DR, editors. Cognitive Informatics for Biomedicine: Human Computer Interaction in Healthcare. 2015

- Zhang J, Walji MF. TURF: Toward a unified framework of EHR usability. J Biomed Inform. 2011 Dec;44(6):1056-1067

- Hettinger AZ, Roth EM, Bisantz AM. Cognitive engineering and health informatics: applications and intersections. J Biomed Inform. 2017;67:21-33

Recorded lectures

- Larry Weed's 1971 Internal Medicine Grand Rounds (https://www.youtube.com/watch?v=qMsPXSMTpFI)

- Selected lectures from the CHOP Department of Biomedical and Health Informatics YouTube channel (https://www. youtube.com/user/chopcbmi1/)

- Selected videos from Workforce Development Programs: Health IT Curriculum Resources for Educators Module 15 (Usability and Human Factors) from the Office of the National Coordinator for Health Information Technology (https://www.healthit. gov/providers-professionals/health-it-curriculum-resources-educators) 